\title{
Tunable Oscillatory Network for Visual Image Segmentation
}

\author{
M.G.Kuzmina ${ }^{1}$, E.A.Manykin ${ }^{2}$ I.I.Surina ${ }^{2}$ \\ ${ }^{1}$ Keldysh Inst. of Appl. Math., Miusskaya Sq. 4, 125047 Moscow, Russia; \\ kuzmina@spp.keldysh.ru \\ 2 RRC Kurchatov Institute, Kurchatov sq. 1, 123182 Moscow, Russia; \\ $\{$ edmany, surina $\} @$ isssph.kiae.ru
}

\begin{abstract}
Recurrent oscillatory network with tunable oscillator dynamics and nonlocal dynamical interaction has been designed. Two versions of the network model have been suggested: 3D oscillatory network of columnar architecture that reflects some image processing features inherent in the brain visual cortex, and $2 \mathrm{D}$ version of the model, obtained from the 3D network by proper reduction.

Image segmentation algorithm developed is based on cluster synchronization of the reduced network that is controlled by means of interaction adaptation method. Our approach provides successive separation of synchronized clusters and final decomposition of the network into a set of mutually desynchronized clusters corresponding to image fragments with different levels of brightness. The algorithm demonstrates the ability of automatical gray-level image segmentation with accurate edge detection. It also demonstrates noise reduction ability.
\end{abstract}

\section{Introduction.}

Since the experimental discovery of synchronous oscillations in the brain visual cortex (VC) of cat and monkey [1,2] the viewpoint was expressed that synchronization-based performance is inherent in VC. Series of attempts was enterprised to elucidate the role of cortical oscillations and synchronization in visual image processing. The hypothesis on the role of dynamical link architecture was initiated since 80th. (C. von der Malsburg, 1983 ; W.Singer et al., 1988). A series of models with various types of oscillators as processing units were designed in the 90th and studied in the context of visual image segmentation problems [3-9] and contour integration task [10].

Bearing in mind a key idea to develop a version of image processing method that would exploit dynamical (instantly rearrangable) connections and synchronization similarly to $\mathrm{VC}$, we designed $3 \mathrm{D}$ oscillatory network of columnar architecture $[13,14]$. Processing units of the network are oscillators formed by pairs of neurons. Internal dynamics of single oscillator is parametrically tunable by two visual image characteristics - local brightness and elementary bar orientation. Besides, there is an important internal parameter of oscillator dynamics - receptive field orientation. Resembling stimulus-dependent response of simple cells of VC, the oscillator can demonstrate either stable oscillatory activity (undamped auto-oscillations) or silence (quickly damped oscillations). Dynamical connec- 
tions in the network are defined in terms of oscillator interaction. They are nonlinearly dependent both on oscillator activities and on receptive field (RF) orientations. Network performance is based on self-controlled synchronization of oscillator ensembles (clusters) encoded by visual image fragments.

$3 \mathrm{D}$ columnar network has been further reduced to $2 \mathrm{D}$ network of idealized oscillator-columns, and image-segmentation algorithm based on clusterized synchronization of the reduced network has been developed. The results of computer simulations demonstrate workability of the algorithm.

\section{Statement of the Problem. 3D Columnar Network}

We try to imitate one step of "top-down" performance of VC: given a visual image in the retina $\mathrm{VC}$ provides image processing via network performance. In our case the performance consists in transfer of recurrent oscillatory network into stable state of clusterized synchronization encoded by visual image fragments.

\subsection{Network Architecture}

Imitating columnar structure of $\mathrm{VC}$ and following [10] we design oscillatory network of columnar architecture consisting of $N^{2}$ columns of $K$ oscillators each so that $N^{2} \cdot K$ is the total number of oscillators. The bases of the columns are located at the nodes of 2D square lattice $G_{N^{2}}$, whereas oscillators of each column are located at the nodes of $1 \mathrm{D}$ lattice $L^{K}$ oriented normally with respect to the plane of $G_{N^{2}}$. Thus, the oscillators of the whole network are located at the nodes of 3D lattice $G_{N^{2}} \times L^{K}$. The state of the 3D network is defined by $(N \times N \times K)$-array of oscillator states $\left[u_{j m}^{k}\right]$. For each oscillator RF orientation is specified by $2 \mathrm{D}$ unit vector $\mathbf{n}_{j m}^{k}$. Vectos $\mathbf{n}_{j m}^{k}$ are supposed deterministically uniformly distributed over the column. The retina is modeled by 2D square lattice similar to $G_{N^{2}}$ so that each retina node corresponds just to one oscillator column.

It is assumed that using continuous distribution of brightness $I(x, y)$ corresponding to retinal image some discretization of $I(x, y)$ is defined in the retina lattice. For our model an matrix of pairs $\left(I_{j m}, \mathbf{s}_{j m}\right), j, m=1, \ldots, N$, should be extracted, where $I_{j m}$ is local value of brightness and $\mathbf{s}_{j m}$ - local orientation of image elementary bar. The $N \times N$ matrix of pairs $\mathcal{M}=\left[\left(I_{j m}, \mathbf{s}_{j m}\right)\right]$ serves further as the set of tuning parameters for oscillatory network.

\subsection{Single Oscillator.}

Following [10], we suppose that single oscillator is formed by a pair of $\mathrm{VC}$ neurons interconnected through excitatory and inhibitory connections. Bearing in mind biologically motivated model of such oscillator, previously proposed by W.Freeman (1987)[11] and Z.Li \& J.Hopfield (1989)[12], we designed limit cycle oscillator with qualitatively similar dynamics and appropriate response to local values $(I, \mathbf{s})$. If oscillator state is defined by a pair of real-valued variables $\left(u_{1}, u_{2}\right)$, the system of ODE for $u_{1}, u_{2}$ can be written in the form of single equation for complex-valued function $u=u_{1}+i \cdot u_{2}$ :

$$
\dot{u}=\left(\rho_{0}^{2}+i \omega-|u-c|^{2}\right)(u-c)+g(I)+q(\mathbf{s}, \mathbf{n}) .
$$


Here $\rho_{0}, c, \omega$ are constants defining asymptotic parameters of the limit cycle of dynamical equation (1). The size (radius $\rho$ ) and location of the limit cycle in the plane $\left(u_{1}, u_{2}\right)$ are controlled by $(I, \mathbf{s})$ via properly constructed functions $g$ and $q$. The following functions $g$ and $q$ are used in eq.(1):

$$
\begin{gathered}
g(I)=1-h\left(I-h_{0}\right), \quad h\left(I-h_{0}\right)=1 /\left(1+e^{-2 \nu\left(I-h_{0}\right)}\right), \\
q(\mathbf{s}, \mathbf{n})=1-\Gamma(|\phi|), \quad \Gamma(|\phi|)=2 e^{-\sigma|\phi|} /\left(1+e^{-2 \sigma|\phi|}\right) .
\end{gathered}
$$

Here $h(x)$ is a continuous step function depending on threshold $h_{0}$, and $\Gamma(|\phi|)$ is narrow symmetrical peak-shaped function depending on the angle $\phi=\beta-\psi$, ehere $\beta$ and $\psi$ are the angles defining orientations of vectors $\mathbf{s}$ and $\mathbf{n}$ respectively: $\mathbf{s}=(\cos \beta, \sin \beta), \quad \mathbf{n}=(\cos \psi, \sin \psi)$.

The limit cycle size $(\rho)$ is sufficiently great if two conditions are satisfied simultaneously: a) $I$ essentially exceeds the threshold value $h_{0}$; b) the angle between $\mathbf{s}$ and $\mathbf{n}$ is sufficiently small. Otherwise, either the limit cycle size is very small, or it degenerates into stable focus.

\subsection{Dynamical Interaction}

Dynamical system governing the dynamics of the network can be written as:

$$
\dot{u}_{j m}^{k}=f\left(u_{j m}^{k}, \mu_{j m}^{k}\right)+S_{j m}^{k} ; \quad j, m=1, \ldots, N, \quad k=1, \ldots, K .
$$

Here $f(u, \mu)=\left(\rho^{2}+i \omega-|u-c|^{2}\right)(u-c)+\mu, \quad \mu_{j m}^{k}=g\left(I_{j m}\right)+q\left(\left|\psi_{j m}^{k}-\beta_{j m}\right|\right)$ are the controlling parameters for oscillator dynamics and the term $S_{j m}^{k}$ specifies oscillator interaction. It has been designed in the form:

$$
S_{j m}^{k}=\sum_{j^{\prime}, m^{\prime}, k^{\prime}} W_{j j^{\prime} m m^{\prime}}^{k k^{\prime}}\left(u_{j m}^{k}, u_{j^{\prime} m^{\prime}}^{k^{\prime}}\right)\left(u_{j^{\prime} m^{\prime}}^{k^{\prime}}-u_{j m}^{k}\right),
$$

where the elements of the matrix of connections $W$ are:

$$
W_{j j^{\prime} m m^{\prime}}^{k k^{\prime}}\left(u, u^{\prime}\right)=P_{j j^{\prime} m m^{\prime}}^{k k^{\prime}}\left(u, u^{\prime}\right) Q_{j j^{\prime} m m^{\prime}}^{k k^{\prime}}\left(\mathbf{n}, \mathbf{n}^{\prime}\right) D_{j j^{\prime} m m^{\prime}}^{k k^{\prime}}\left(\left|\mathbf{r}-\mathbf{r}^{\prime}\right|\right),
$$

where $\mathbf{n}$ and $\mathbf{n}^{\prime}$ are RF orientations, $\quad \mathbf{r}$ and $\mathbf{r}^{\prime}$ are radius-vectors, defining spatial locations of oscillators $(j, m, k)$ and $\left(j^{\prime}, m^{\prime}, k^{\prime}\right)$ in the network. Three cofactors introduced in the expression for $W_{j j^{\prime} m m^{\prime}}^{k k^{\prime}}$ provide the following features of dynamical interaction: a) dependence on oscillatory activity; b) dependence on RF orientations; c) dependence on spatial distance between the oscillators in the network.

The cofactors $P_{j j^{\prime} m m^{\prime}}^{k k^{\prime}}$ providing the dependence of oscillator interaction on oscillatory activity are chosen in the form:

$$
P_{j j^{\prime} m m^{\prime}}^{k k^{\prime}}=w_{0} \cdot\left\langle\left(\rho_{j m}^{k} \rho_{j^{\prime} m^{\prime}}^{k^{\prime}}-h\right)\right.
$$

where $\rho_{j m}^{k}$ is limit cycle radius of the oscillator defined by indices $(j, m, k)$, $\langle(x)$ is continuous step function similar to that one in eq.(2), $h$ is a threshold, 
$w_{0}$ is a constant of interaction. The cofactors $Q_{j j^{\prime} m m^{\prime}}^{k k^{\prime}}$ providing the dependence on RF orientations are defined as $Q_{j j^{\prime} m m^{\prime}}^{k k^{\prime}}=\Gamma\left(\left|\psi_{j m}^{k}-\psi_{j^{\prime} m^{\prime}}^{k^{\prime}}\right|\right)$, where $\Gamma(|\phi|)$ is introduced in eq.(3). At last, the cofactors $D_{j j^{\prime} m m^{\prime}}^{k k^{\prime}}$, providing spatially nonlocal character of network interaction, can be defined by any function vanishing at some finite (variable) spatial distance.

\section{Reduced Oscillatory Network}

The 3D columnar oscillatory network can be naturally reduced to its limit version - 2D network defined in the lattice $G_{N^{2}}$ and consisting of idealized oscillatorcolumns as processing units. The reduced network can be obtained via intercolumn averaging of oscillator response and special limit analogous to well-known thermodynamical limit in statistical physics. As a result, RF orientations dissapear from consideration at all, and internal dynamics of oscillator-column, governed by eq.(1) with $q(\mathbf{s}, \mathbf{n}) \equiv 0$, depends on single image characteristics - local brightness $I$. The state of the network is defined by $N \times N$ matrix $\hat{u}=\left[u_{j m}\right]$, and network dynamical equations are:

$\dot{u}_{j m}=\left(\rho^{2}+i \omega_{j m}-\left|u_{j m}-c\right|^{2}\right)\left(u_{j m}-c\right)+g\left(I_{j m}\right)=\sum_{j^{\prime}, m^{\prime}=1}^{N} W_{j m j^{\prime} m^{\prime}}\left(u_{j^{\prime} m^{\prime}}-u_{j m}\right)$,

where

$$
W_{j j^{\prime} m m^{\prime}}=P_{j j^{\prime} m m^{\prime}}\left(u, u^{\prime}\right) D_{j j^{\prime} m m^{\prime}}\left(\left|\mathbf{r}-\mathbf{r}^{\prime}\right|\right),
$$

The cofactors $P_{j j^{\prime} m m^{\prime}}$ and $D_{j j^{\prime} m m^{\prime}}$ in eq.(9) are calculated in the same manner as in the case of $3 \mathrm{D}$ network, but the cofactor $Q$ is now reduced to 1 . We are able to consider further the reduced network as independent 2D model and develop an image segmentation algorithm based on clusterized synchronization of the network.

\section{Interaction Adaptation Method. Simulation Results}

We introduce new additional controlling parameter $\gamma$ which will allow to improve algorithm performance due to sequential excluding of synchronized clusters from network interaction. Namely, we introduce modified dynamical connections in 2D network

$$
\tilde{W}_{j j^{\prime} m m^{\prime}}=W_{j j^{\prime} m m^{\prime}} \Gamma\left(\gamma_{j m}-\gamma_{j^{\prime} m^{\prime}}\right),
$$

where $\Gamma\left(\gamma-\gamma^{\prime}\right)$ is the function defined in eq. (3), and use the following interaction adaptation method. Let an image to be segmented be defined by piecewise constant martrix $\left[I_{j m}\right]$, where $I_{j m}$ belong to some quantized scale of brightness $\mathcal{I}=\left\{I^{(l)}\right\}, \quad I^{(1)}>I^{(2)}>\ldots>I^{(L)}$. Put at initial step $\gamma_{j m} \equiv 0$ in the matrix of controlling parameters $\tilde{\mathcal{M}}=\left[I_{j m}, \gamma_{j m}\right]$. We realize a process of interaction strengthening via gradual decreasing of threshold $h$ in eq.(7), starting from sufficiently high initial value at which the network is totally desynchronized. One can observe the following sequence of network states. At some value of $h=h_{1}$ the first synchronized cluster, corresponding to image fragment of brightness $I^{(1)}$, arises whereas the rest network remains desynchronized. Such network 
state is conserved at $h \in\left[h_{2}, h_{1}\right]$. It provides the possibility of separation of the first cluster via excluding it from network interaction. In view of eq. (10) excluding from interaction can be achieved simply by prescribing some nonzero value $\gamma=\gamma_{1}, \quad \gamma_{1} \geq b>0$, to those components of the matrix $\tilde{\mathcal{M}}$ that correspond to spatial locations of oscillators belonging to the synchronized cluster. Further continuation of interaction strengthening provides analogous separation of the second synchronized cluster and so on. The process is repeated until all the clusters corresponding to the scale $\mathcal{I}$ will be successively synchronized and separated. In final state the network will be decomposed into the set of mutually desynchronized clusters. The accurate detection of boundaries between image fragments occurs. It is the direct consequence of the essential attribute of oscillator dynamics - sharp dependence of limit cycle size on local image brightness. Several synthetic images were used for testing the algorithm. Three stages of algorithm performance are shown in Fig.1.

\section{Summary}

Recurrent oscillatory network of columnar architecture for visual image processing has been designed. Internal dynamics of single oscillator is parametrically tunable by visual image characteristics - local brightness and bar orientation. Nonlocal dynamical connections are dependent on oscillatory activities and receptive field orientations. 2D reduced oscillatory network has been extracted. Image segmentation algorithm has been developed based on interaction adaptation for $2 \mathrm{D}$ network. The algorithm provides successive separation of synchronized network clusters and demonstrates promising advantages: clearly observable image segmentation, accurate edge detection and noise reduction.

\section{References}

[1] Eckhorn R. Bauer R., Jordan W., Brosch M., Kruse W., Munk M., Reitboek H.J.: Biol. Cybern., 60, (1988), 121-130.

[2] Gray C.M., Singer W.: Proc. Natl. Acad. Sci. USA, 86, (1989), 1698-1702.

[3] Schuster H.C., Wagner P.: Biol. Cyb., 64, (1990), 77-85.

[4] Wang D., Buhman J., von der Malsburg C.: Neur. Comp., 2, (1990), 94-106.

[5] König P., Schillen T.B.: Neur. Comp., 3, (1991), 155-166.

[6] Sompolinsky H., Golomb D., Kleinfeld D.: Phys. Rev. A, 43, (1991), 69907011.

[7] Wang D.L., Terman D.: IEEE Trans. NN, 6, (1995), 283-286.

[8] Wang D.L., Terman D.: Neur. Comp., 9, (1997), 805-836.

[9] Chen K., Wang D.L., Liu X.: IEEE Trans. NN, 11, (2000), 1106-1123.

[10] Li Z.: Neur. Comp., 10, (1998), 903-940.

[11] Freeman W.J.: Electroenceph. Clin. Neurophys., 44, (1978), 586-605.

[12] Li Z., Hopfield J.J.: Biol. Cybern., 61(5), (1989), 379-392.

[13] Kuzmina M.G., Surina I.I.: Proc. of AICS'99, Cork, (1999), 37-43.

[14] Kuzmina V.G., Surina I.I.: Proc. of NOLTA'2000, Dresden, Vol.1, (2000), $335-338$. 